\title{
Effects of atorvastatin in combination with ezetimibe on carotid atherosclerosis in elderly patients with hypercholesterolemia
}

\author{
P. Luo ${ }^{1,2}$, L. Li ${ }^{1}$, L.X. Wang ${ }^{3}$, H.H. Zhu ${ }^{4}$, S. Du ${ }^{3}$, S.L. Wu ${ }^{3}$, Y.G. Han ${ }^{3}$ and \\ G.G. Wang ${ }^{3}$ \\ ${ }^{1}$ Department of Cardiology, Qilu Hospital of Shandong University, \\ Jinan, China \\ ${ }^{2}$ Department of Cardiology, Zhengzhou University People's Hospital, \\ Zhengzhou, China \\ ${ }^{3}$ Department of Cardiology, Henan Provincial People's Hospital, \\ Zhengzhou, China \\ ${ }^{4}$ Department of Ultrasound, Henan Provincial People's Hospital, \\ Zhengzhou, China \\ Corresponding author: L. Li \\ E-mail: LiLidoccn@126.com
}

Genet. Mol. Res. 13 (2): 2377-2384 (2014)

Received June 10, 2013

Accepted January 13, 2014

Published April 3, 2014

DOI http://dx.doi.org/10.4238/2014.April.3.10

\begin{abstract}
The aim of this study was to observe the effects of atorvastatin combined with ezetimibe on carotid atherosclerosis in elderly patients with hypercholesterolemia. A total of 84 elderly hypercholesterolemic patients complicated with carotid atherosclerosis were divided into control group (atorvastatin alone) and combined group (atorvastatin combined with ezetimibe) and treated for 12 months. Carotid atherosclerosis-related indicators including blood lipid and high-sensitivity C-reactive protein (hsCRP) were determined before and after treatment. The levels of carotid intima-media thickness (CIMT), serum low density lipoprotein cholesterol (LDL-C) and hsCRP were markedly decreased $(\mathrm{P}<0.05)$ after treatment in the
\end{abstract}


two groups, while the reduction of the levels of CIMT, serum LDL-C and hsCRP was more significant in the combined group $(\mathrm{P}<0.01)$. After treatment, the levels of CIMT, serum LDL-C and hsCRP were distinctly different between combined and control group $(\mathrm{P}<0.05)$. The combination of atorvastatin with ezetimibe could further decrease LDL-C and hsCRP levels and have certain effects on the progression of carotid atherosclerosis in elderly patients with hypercholesterolemia.

Key words: Ezetimibe; Atorvastatin; Carotid atherosclerosis; Hypercholesterolemia

\section{INTRODUCTION}

A reduction in low density lipoprotein cholesterol (LDL-C) by at least 30 to $50 \%$ can clinically benefit patients at high risk of coronary heart disease (CHD) and related diseases (Benge et al., 2012), but it is difficult to achieve this with atorvastatin alone. Recent clinical research has shown that atorvastatin in combination with cholesterol absorption inhibitor ezetimibe significantly reduces LDL-C level in patients with hypercholesterolemia, showing a superior lipid-lowering efficacy compared to statin alone (Roeters van Lennep et al., 2008), but whether the distinct decrease in LDL-C level by the combination of atorvastatin and ezetimibe can slow down or reverse the process of atherosclerosis is still inconclusive. In our research, we explored whether a more substantial reduction in LDL-C level could further ameliorate carotid atherosclerosis through comparing the effects of atorvastatin in combination with ezetimibe and atorvastatin alone on LDL-C, high-sensitivity C-reactive protein (hsCRP) and carotid intima-media thickness (CIMT).

\section{MATERIAL AND METHODS}

\section{Subjects}

A total of 84 elderly hypercholesterolemic patients, including 44 males and $40 \mathrm{fe}-$ males with an average age of $(67.23 \pm 5.64)$ years were selected in our department between July 2010 and December 2011. Among these patients, 70 of them were confirmed with CHD by coronary arteriography, 28 patients were diagnosed with type 2 diabetes according to criteria for the diagnosis and classification of diabetes published by WHO in 1999, and 10 cases were diabetes complicated with CHD. The patients with hypertension, blood diseases, hepatorenal dysfunction, severe infectious disease and heart failure were excluded. All the selected patients who still had abnormal LDL-C levels $(\leq 2.6 \mathrm{mM})$ after undergoing lipid-lowering therapy for three months were randomized into control and combined groups. No differences in age, gender, body mass index, blood pressure, and smoking were observed in patients between the two groups (Table 1).

\section{Methods}

The control and combined groups used atorvastatin alone (Lipitor, $20 \mathrm{mg} / \mathrm{night}$, 
Pfizer, Inc.) and atorvastatin in combination with ezetimibe (Zetia, $10 \mathrm{mg} /$ day, Merck Sharp \& Dohme) for 12 months, respectively. Hepatorenal function, myocardial enzyme and glycosylated hemoglobin were monitored during drug application. Glycosylated hemoglobin level in patients with diabetes was controlled within 7\%. CIMT, carotid plaque Crouse integral, carotid diameter, blood lipid level and hsCRP were detected before and after treatment. CIMT measurement: CIMT was detected three times $10 \mathrm{~mm}$ away from the bifurcation of bilateral common carotid artery and average was calculated; $0.9 \mathrm{~mm} \leq \mathrm{IMT}<1.3 \mathrm{~mm}$ was defined as thickening and IMT $\geq 1.3 \mathrm{~mm}$ refers to plaque. Carotid plaque Crouse integral measurement: maximum thickness $(\mathrm{mm})$ of the isolated plaques in bilateral carotid artery was added regardless of length of various plaques. Blood lipid level and hsCRP were determined with a Hitachi 7060 automatic biochemistry analyzer and glycosylated hemoglobin was determined by affinity chromatography.

\begin{tabular}{|c|c|c|}
\hline Items & Control group $(\mathrm{N}=44)$ & Combined group $(\mathrm{N}=40)$ \\
\hline Gender (male/female, cases) & $22 / 22$ & $22 / 18$ \\
\hline Age (years) & $66.31 \pm 5.82$ & $67.21 \pm 6.40$ \\
\hline Body mass index & $24.72 \pm 4.42$ & $24.43 \pm 4.61$ \\
\hline \multicolumn{3}{|l|}{ Etiological factor } \\
\hline CHD (cases) & 34 & 36 \\
\hline Diabetes (cases) & 16 & 12 \\
\hline Systolic pressure (mmHg) & $132.52 \pm 15.43$ & $133.52 \pm 15.24$ \\
\hline Diastolic pressure (mmHg) & $66.31 \pm 10.52$ & $65.45 \pm 9.62$ \\
\hline
\end{tabular}

$1 \mathrm{mmHg}=0.133 \mathrm{kPa}$; CHD $=$ coronary heart disease.

\section{Statistical analysis}

Data were analyzed with SPSS12.0 software and measurement data are reported as means $\pm \mathrm{SD}$. Comparison before and after treatment within groups was done with a $t$-test and comparison between groups by variance analysis. $\mathrm{P}<0.05$ was considered to be statistically significant

\section{RESULTS}

\section{Comparison of blood lipid and hsCRP before and after treatment between two groups}

The levels of serum LDL-C, triglycerides and hsCRP in the two groups distinctly declined after 12-months treatment compared with levels before treatment, while the serum high density lipoprotein cholesterol (HDL-C) level markedly increased $(\mathrm{P}<0.05$, Table 2$)$. The reduction in the levels of CIMT, serum LDL-C and hsCRP was more significant in the combined group $(\mathrm{P}<0.01$, Table 2$)$. After treatment, the levels of serum LDL-C and hsCRP were significantly lower in the combined group than those in the control group $(\mathrm{P}<0.05$, Table 2). Serum HDL-C increased and triglycerides decreased after 12-months treatment in the combined group compared with the control group, but these changes were not statistically significant. 
Table 2. Comparison of blood lipid and hs-CRP before and after treatment between two groups (means \pm SD).

\begin{tabular}{|c|c|c|c|c|}
\hline & \multicolumn{2}{|c|}{ Control group $(\mathrm{N}=44)$} & \multicolumn{2}{|c|}{ Combined group $(\mathrm{N}=40)$} \\
\hline & Before treatment & $\overline{\text { After treatment }}$ & Before treatment & After treatmen \\
\hline $\mathrm{TC}(\mathrm{mM})$ & $5.88 \pm 1.42$ & $5.16 \pm 1.34 *$ & $5.75 \pm 1.58$ & $4.95 \pm 1.43^{*}$ \\
\hline LDL-C (mM) & $3.31 \pm 0.46$ & $2.75 \pm 0.58 *$ & $3.27 \pm 0.36$ & $2.31 \pm 0.54 * * \Delta$ \\
\hline HDL-C (mM) & $1.18 \pm 0.46$ & $1.34 \pm 0.41^{*}$ & $1.17 \pm 0.38$ & $1.36 \pm 0.22 *$ \\
\hline $\mathrm{TG}(\mathrm{mM})$ & $2.35 \pm 0.64$ & $1.78 \pm 0.45^{*}$ & $2.28 \pm 0.48$ & $2.15 \pm 0.64 *$ \\
\hline hsCRP (mg/L) & $7.15 \pm 2.43$ & $5.08 \pm 1.64 *$ & $7.09 \pm 2.68$ & $4.36 \pm 1.23 * * \Delta$ \\
\hline
\end{tabular}

Compared with that before treatment, ${ }^{*} \mathrm{P}<0.05, * * \mathrm{P}<0.01$; compared with control group after treatment, ${ }^{\Delta} \mathrm{P}<0.05$.

$\mathrm{TC}=$ total cholesterol; LDL-C $=$ low density lipoprotein cholesterol; HDL-C $=$ high density lipoprotein cholesterol;

$\mathrm{TG}=$ triglyceride; hsCRP $=$ high-sensitivity $\mathrm{C}$-reactive protein .

\section{Comparison of CIMT, carotid plaque Crouse integral and carotid inner diameter before and after treatment between two groups}

No differences in various indicators were observed before treatment in the two groups. CIMT and carotid plaque Crouse integral dramatically decreased, and carotid inner diameter increased notably after 12-months treatment compared with that before treatment in both the control $(\mathrm{P}<0.05$, Table 3$)$ and combined $(\mathrm{P}<0.01$, Table 3$)$ groups, where the reduction in CIMT and carotid plaque Crouse integral was more significant in the combined group. After treatment, the levels of CIMT and carotid plaque Crouse integral were significantly lower in the combined group than in the control group. The change in CIMT before and after treatment in the two groups is shown in Figures 1-4: Figure 1 represents the control group before treatment, where CIMT was $1.2 \mathrm{~mm}$; Figure 2 represents the control group after one year of treatment, where CIMT decreased to $1.1 \mathrm{~mm}$; Figure 3 represents the combined group before treatment, where CIMT was $1.4 \mathrm{~mm}$; Figure 4 represents the combined group after one year of treatment, where CIMT was $1.2 \mathrm{~mm}$.

Table 3. Comparison of carotid intima-media thickness (CIMT) and plaque Crouse integral before and after treatment between two groups (means $\pm \mathrm{SD}$ ).

\begin{tabular}{|c|c|c|c|c|}
\hline & \multicolumn{2}{|c|}{ Control group $(\mathrm{N}=44)$} & \multicolumn{2}{|c|}{ Combined group $(\mathrm{N}=40)$} \\
\hline & Before treatment & After treatment & Before treatment & After treatment \\
\hline CIMT (mm) & $1.23 \pm 0.25$ & $1.12 \pm 0.14^{*}$ & $1.26 \pm 0.24$ & $1.02 \pm 0.11^{* * \Delta}$ \\
\hline Plaque crouse integral & $4.27 \pm 1.53$ & $4.02 \pm 1.24 *$ & $4.28 \pm 1.61$ & $3.78 \pm 1.22^{* * \Delta}$ \\
\hline Carotid diameter $(\mathrm{mm})$ & $6.08 \pm 0.35$ & $6.28 \pm 0.47 *$ & $6.10 \pm 0.31$ & $6.40 \pm 0.39 *$ \\
\hline
\end{tabular}

Compared with that before treatment, ${ }^{*} \mathrm{P}<0.05,{ }^{* *} \mathrm{P}<0.01$; compared with control group after treatment, ${ }^{\mathrm{A}}<0.05$.

\section{Comparison of cardiovascular events and safety}

There were no events of myocardial infarction and cardiovascular death during a 12-month follow-up. One patient in the control group had a transient elevation in glutamicpyruvic transaminase and glutamic oxalacetic transaminase levels, but they were lower than three times the upper limit of normal, which recovered back to the normal range after application of atorvastatin; creatine kinase was not unusually high; there were no myalgia, rashes or other clinical adverse reactions. 


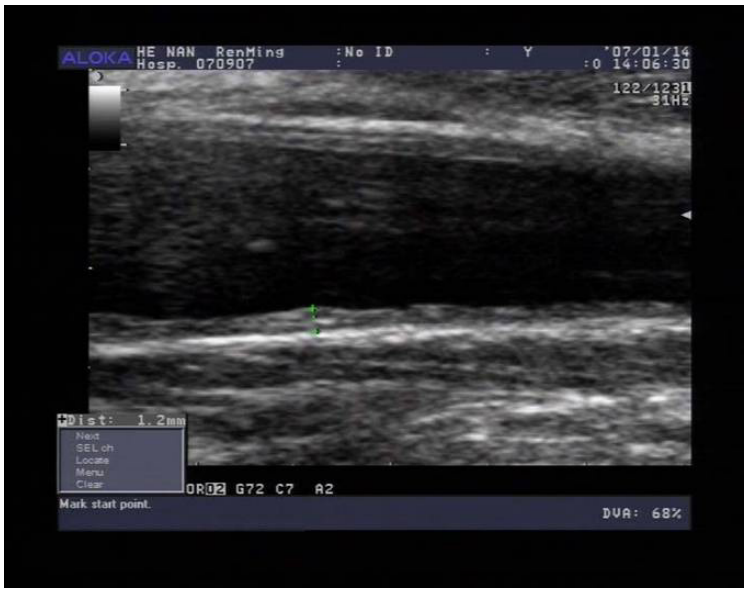

Figure 1. Before treatment in control group.

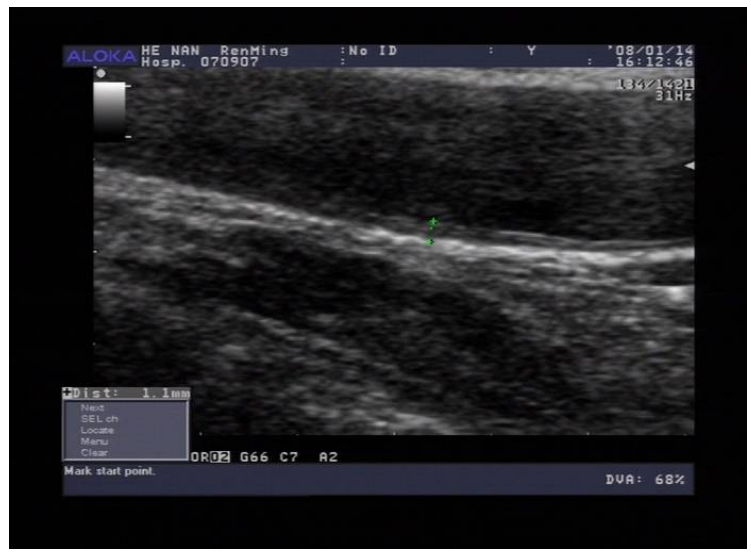

Figure 2. After treatment in control group.

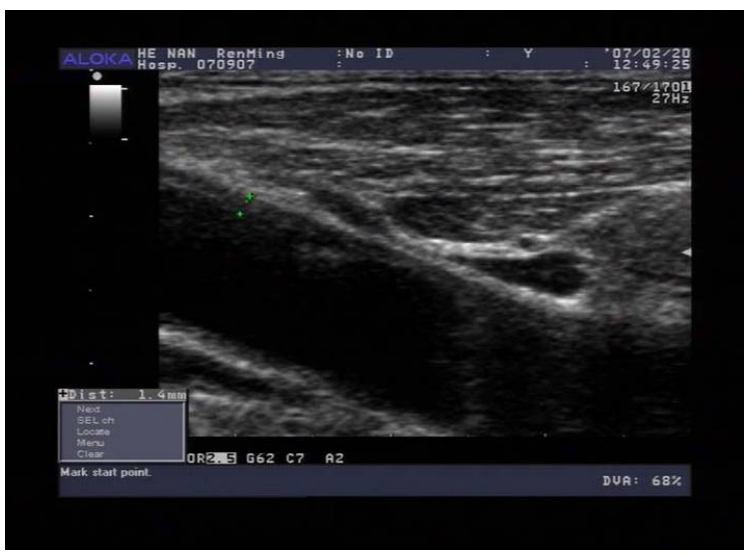

Figure 3. Before treatment in combined group. 


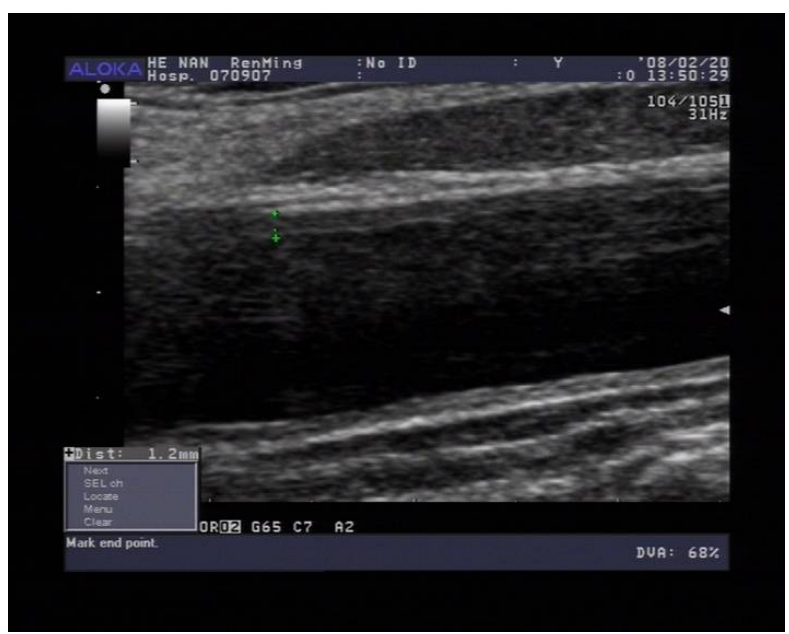

Figure 4. After treatment in combined group.

\section{DISCUSSION}

Intensive lipid-lowering is an important measure for primary and secondary prevention of cardiovascular disease. It is more important for patients at high risk or very high risk of cardiovascular event to receive statin treatment to reduce LDL-C levels back to normal or by 30 to $50 \%$. However, the initial dose of the statins can reduce LDL-C level by 20 to $30 \%$, and doubling the dose only provides a 5 to $6 \%$ further decrease (Wierzbicki and Mikhailidis, 2002). On the other side, it is very dangerous to increase the statin dose without paying attention to drug safety. Ezetimibe is a novel cholesterol-lowering drug, which decreases lipid levels by selectively inhibiting the absorption of cholesterol by brush border of jejunum mucosa (Clearfield, 2003). The combination of statin and ezetimibe stops both the synthesis and absorption of cholesterol so as to synergistically reduce LDL-C. Many clinical trials have demonstrated that statin combined with ezetimibe significantly reduces the levels of LDL$\mathrm{C}$ and hsCRP, which is superior to statin alone in lipid-lowing efficacy (Stein et al., 2004; Ballantyne et al., 2007; Okada et al., 2012). A recent study has confirmed that ezetimibe not only decreases cholesterol absorption but also affects the process of atherosclerosis by inhibiting Rho kinase activity (Nochioka et al., 2012). Thus, the combination is able to reduce major atherosclerotic events and all-cause mortality (Hayek et al., 2013).

Atherosclerosis is the most common and important pathologic change in cardiovascular disease that causes disability and death. The increase in CIMT is a noninvasive indicator to evaluate early stages of atherosclerosis, as well as an important hazard and predictive factor of cardiovascular disease (Robertson et al., 2012). Extensive research has confirmed that the incidence of cardiovascular events increases when CIMT is elevated in type 2 diabetic patients (Irie et al., 2012). Keo et al. (2011) found that CIMT closely correlates with ischemic events in patients with atherosclerotic vascular disease. Evidence-based medicine has shown that lipid-lowering to normal levels is always accompanied by the vanishing, delayed development or stopping of atherosclerotic plaque. When comparing atorvastatin and simvastatin for their effect on the development of atherosclerosis in the ASAP study, it was discovered that a large 
dose of atorvastatin could reduce LDL-C level as well as CIMT level (Smilde et al., 2001). Data analysis from the SANDS study revealed that the application of simvastatin or simvastatin plus ezetimibe could reduce the LDL-C level in type 2 diabetic patients over 40 years old, and there was a positive correlation between the improvement degree of carotid artery atherosclerosis and the extent of LDL-C reduction (Fleg et al., 2008). hsCRP is an important proinflammatory factor that is closely related to atherosclerosis occurrence and development. A to $\mathrm{Z}$ research and PROVE IT trials showed that the patients with a low level of hsCRP had a better prognosis and that the fatality rate of patients with high level of hsCRP rose exponentially (Morrow et al., 2006; Scirica et al., 2009). Some research has revealed that hsCRP is independently associated with cardiovascular events in the patients with arteriosclerosis and arterial occlusive disease (Kablak-Ziembicka et al., 2011). Numerous studies have confirmed that hsCRP level markedly declines and that vascular endothelial function apparently improves along with the decrease in LDL-C (Heart Protection Study Collaborative Group et al., 2011; Grigore et al., 2013).

Our research revealed that the levels of serum LDL-C, triglycerides and hsCRP in patients were markedly decreased, CIMT and carotid plaque Crouse integral clearly ameliorated, and the coronary inner diameter distinctly increased after atorvastatin treatment or atorvastatin in combination with ezetimibe, while the reduction in serum LDL-C and hsCRP was more significant and the decrease in CIMT and carotid plaque Crouse integral was greater in the combined group. After treatment, serum LDL-C and hsCRP levels, as well as CIMT and carotid plaque Crouse integral were statistically lower in the combined group versus control group. These results indicated that the combination of atorvastatin with ezetimibe had stronger hypolipidemic and anti-inflammatory effects, simultaneously further improved CIMT and carotid plaque Crouse integral, and slowed down the progression of carotid atherosclerosis in elderly patients with hypercholesterolemia. In addition, the hsCRP level in patients was slightly high in our study, which was considered to correlate with $\mathrm{CHD}$, arteriosclerosis, diabetes, hypercholesterolemia, etc.

However, whether the distinct decrease in serum LDL-C level by the combination of atorvastatin with ezetimibe can slow down or reverse the process of atherosclerosis is still controversial. The ENHANCE group randomized 720 patients with heterozygous hypercholesterolemia into an atorvastatin in combination with ezetimibe group and atorvastatin group, but there was no significant difference in CIMT change between two groups after two-years of treatment (Kastelein et al., 2008). Most authors believe that the ENHANCE study did not set the CIMT baseline, which led to the negative result; this research can not affect current clinical intensive lipid-lowering strategy (Gandhi, 2008), and large studies on atorvastatin in combination with ezetimibe are being conducted with clinical endpoint events for this purpose. The long-term efficacy of ezetimibe requires further investigation.

In conclusion, the combination of atorvastatin with ezetimibe could further decrease serum LDL-C and hsCRP levels so as to ameliorate CIMT. However, whether intensive lipidlowering can slow down or reverse the process of atherosclerosis is still controversial, which requires scientific large-scale clinical trials to confirm this.

\section{REFERENCES}

Ballantyne CM, Weiss R, Moccetti T, Vogt A, et al. (2007). Efficacy and safety of rosuvastatin $40 \mathrm{mg}$ alone or in combination with ezetimibe in patients at high risk of cardiovascular disease (results from the EXPLORER study). Am. J. Cardiol. 99: 673-680.

Benge CD, Markley B and McFarland MS (2012). Role of aggressive LDL reduction in patients with coronary heart disease. South Med. J. 105: 48-55. 
Clearfield MB (2003). A novel therapeutic approach to dyslipidemia. J. Am. Osteopath. Assoc. 103: S16-S20.

Fleg JL, Mete M, Howard BV, Umans JG, et al. (2008). Effect of statins alone versus statins plus ezetimibe on carotid atherosclerosis in type 2 diabetes: the SANDS (Stop Atherosclerosis in Native Diabetics Study) trial. J. Am. Coll. Cardiol. 52: 2198-2205.

Gandhi MJ (2008). Does 'ENHANCE' diminish confidence in ezetimibe? J. Assoc. Physicians India 56: 665-666.

Grigore L, Raselli S, Garlaschelli K, Redaelli L, et al. (2013). Effect of treatment with pravastatin or ezetimibe on endothelial function in patients with moderate hypercholesterolemia. Eur. J. Clin. Pharmacol. 69: 341-346.

Hayek S, Canepa EF, Sattar A, Gamalski S, et al. (2013). Effect of ezetimibe on major atherosclerotic disease events and all-cause mortality. Am. J. Cardiol. 111: 532-539.

Irie Y, Katakami N, Kaneto H, Kasami R, et al. (2012). Maximum carotid intima-media thickness improves the prediction ability of coronary artery stenosis in type 2 diabetic patients without history of coronary artery disease. Atherosclerosis 221: 438-444.

Heart Protection Study Collaborative Group, Jonathan E, Derrick B, Emma L, et al. (2011). C-reactive protein concentration and the vascular benefits of statin therapy: an analysis of 20,536 patients in the Heart Protection Study. Lancet 377 : 469-476.

Kablak-Ziembicka A, Przewlocki T, Sokolowski A, Tracz W, et al. (2011). Carotid intima-media thickness, hsCRP and TNF- $\alpha$ are independently associated with cardiovascular event risk in patients with atherosclerotic occlusive disease. Atherosclerosis 214: 185-190.

Kastelein JJ, Akdim F, Stroes ES, Zwinderman AH, et al. (2008). Simvastatin with or without ezetimibe in familial hypercholesterolemia. N. Engl. J. Med. 358: 1431-1443.

Keo HH, Baumgartner I, Hirsch AT, Duval S, et al. (2011). Carotid plaque and intima-media thickness and the incidence of ischemic events in patients with atherosclerotic vascular disease. Vasc. Med. 16: 323-330.

Morrow DA, de Lemos JA, Sabatine MS, Wiviott SD, et al. (2006). Clinical relevance of C-reactive protein during followup of patients with acute coronary syndromes in the Aggrastat-to-Zocor Trial. Circulation 114: 281-288.

Nochioka K, Tanaka S, Miura M, Zhulanqiqige dE, et al. (2012). Ezetimibe improves endothelial function and inhibits Rho-kinase activity associated with inhibition of cholesterol absorption in humans. Circ. J. 76: 2023-2030.

Okada K, Iwahashi N, Endo T, Himeno H, et al. (2012). Long-term effects of ezetimibe-plus-statin therapy on low-density lipoprotein cholesterol levels as compared with double-dose statin therapy in patients with coronary artery disease. Atherosclerosis 224: 454-456.

Robertson CM, Gerry F, Fowkes R and Price JF (2012). Carotid intima-media thickness and the prediction of vascular events. Vasc. Med. 17: 239-248.

Roeters van Lennep HW, Liem AH, Dunselman PH, Dallinga-Thie GM, et al. (2008). The efficacy of statin monotherapy uptitration versus switching to ezetimibe/simvastatin: results of the EASEGO study. Curr. Med. Res. Opin. 24: 685-694.

Scirica BM, Cannon CP, Sabatine MS, Jarolim P, et al. (2009). Concentrations of C-reactive protein and B-type natriuretic peptide 30 days after acute coronary syndromes independently predict hospitalization for heart failure and cardiovascular death. Clin. Chem. 55: 265-273.

Smilde TJ, van Wissen S, Wollersheim H, Trip MD, et al. (2001). Effect of aggressive versus conventional lipid lowering on atherosclerosis progression in familial hypercholesterolaemia (ASAP): a prospective, randomised, double-blind trial. Lancet 357: 577-581.

Stein E, Stender S, Mata P, Sager P, et al. (2004). Achieving lipoprotein goals in patients at high risk with severe hypercholesterolemia: efficacy and safety of ezetimibe co-administered with atorvastatin. Am. Heart J. 148: 447-455.

Wierzbicki AS and Mikhailidis DP (2002). Dose-response effects of atorvastatin and simvastatin on high-density lipoprotein cholesterol in hypercholesterolaemic patients: a review of five comparative studies. Int. J. Cardiol. 84: 53-57. 